Klaus Richter

\title{
Semiclassical Theory of Mesoscopic Quantum Systems
}

With 50 Figures 


\section{Contents}

1. Introduction $\ldots \ldots \ldots \ldots \ldots \ldots \ldots \ldots \ldots \ldots \ldots \ldots \ldots \ldots$

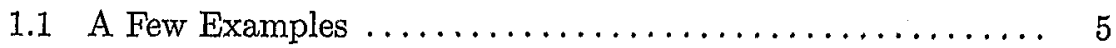

1.1.1 Antidot Superlattices . ............... 5

1.1.2 Ballistic Weak Localization in Electron Billiards . . . . . 8

1.1.3 Mesoscopic Orbital Magnetism .............. 11

1.1.4 Andreev Billiards $\ldots \ldots \ldots \ldots \ldots \ldots \ldots \ldots \ldots \ldots \ldots, 12$

1.1.5 Quantum Corrals..................... 13

1.2 Purpose of This Book and Overview ............ 15

2. Elements of Modern Semiclassical Theory ............. 19

2.1 Green Functions and Trace Formulas................. 19

2.1.1 Semiclassical Green Function .............. 20

2.1 .2 Density of States .................... 24

2.1.3 Berry-Tabor Trace Formula ............. 25

2.1.4 Gutzwiller Trace Formula ................ 26

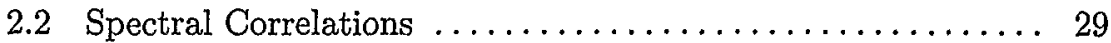

2.3 Thermodynamic Quantities ................... 32

2.4 Semiclassical Linear Response ................. 35

2.4.1 Basic Quantum Mechanical Relations ........... 36

2.4.2 Semiclassical Approximation: Overview .......... 39

2.4 .3 Chaotic Case ...................... 40

2.4 .4 Integrable Case $\ldots \ldots \ldots \ldots \ldots \ldots \ldots \ldots \ldots \ldots \ldots . \ldots \ldots$

2.4.5 Dynamic Susceptibilities ............... 46

3. Ballistic Quantum Transport ................ 49

3.1 Bulk Conductivity . ...................... 51

3.1.1 Semiclassical Approach.................. 51

3.1.2 Antidot Lattices: Experiments . ........... 55

3.1.3 Antidot Lattices: Conductivity Calculations ........ 56

3.2 Transport Through Phase-Coherent Conductors .......... 62

3.2.1 Semiclassical Approach to Landauer Conductance .... 63

3.2 .2 Trajectory Analysis . . . . . . . . . . . . . . 66

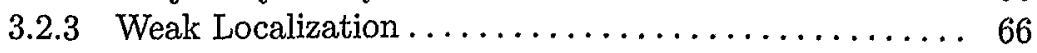

3.2.4 Finite Antidot Arrays . . . . . . . . . . . . . 68 
3.2.5 Conductance Fluctuations . . . . . . . . . . . . . 71

3.3 Limitations of Present Semiclassical Transport Theory ...... 73

4. Orbital Magnetism .......................... 79

4.1 Historical Backround and Overview ................ 79

4.2 Basic Concepts............................. 83

4.2.1 Definitions . ......................... 83

4.2.2 Bulk Properties: De Haas-van Alphen Effect and Landau Diamagnetism ................. 84

4.2.3 Thermodynamics in the Mesoscopic Regime ....... 87

4.3 Chaotic Systems ........................... 90

4.3.1 Semiclassical Approaches .................... 91

4.3.2 Persistent Currents ......................... 94

4.3.3 Magnetic Susceptibilities ..................... 99

4.3.4 Systems with Diffusive Dynamics ................ 104

4.3.5 Relation to Experiments ..................... 104

4.4 Perturbed Integrable Systems: General Framework......... 105

4.4.1 Magnetic Susceptibility ................... 107

4.4.2 Integrable Versus Chaotic Behavior .............. 109

4.5 Perturbed Integrable Systems: Square Quantum Wells....... 110

4.5.1 The Weak-Field Density of States .............. 111

4.5.2 Susceptibility of Individual Samples

and Ensemble Averages ..................... 114

4.5.3 Bouncing-Ball Magnetism .................... 121

4.5.4 De Haas-van Alphen-Like Oscillations . .......... 126

4.6 Systems Integrable at Arbitrary Fields: Ring Geometries . . . 128

4.6.1 Persistent Currents .......................... 129

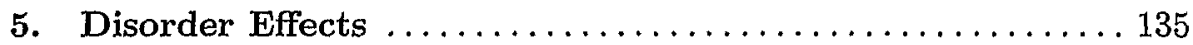

5.1 Characterization ............................ 135

5.2 Semiclassical Treatment of Disorder in the Ballistic Limit.... 138

5.2.1 The Disorder Model ...................... 138

5.2.2 Effect on the Single-Particle Green Function ....... 139

5.2.3 Effect on the Two-Particle Green Function .......... 143

5.3 High Landau Levels in a Smooth Disorder Potential ........ 144

5.4 Magnetic Susceptibility of Ballistic Quantum Dots ........ 147

5.4.1 Fixed-Size Impurity Average ................. 147

5.4 .2 Combined Impurity and Size Average ............ 150

5.4 .3 Concluding Remarks . . . . . . . . . . . . . . . . . . . . . 154

5.5 From Ballistic to Diffusive Dynamics ................ 155

5.5.1 Spectral Correlations in the Diffusive Limit ........ 156

5.5.2 Spectral Correlations

in Disordered Nondiffusive Systems ............. 156

5.5 .3 Orbital Magnetism ....................... 161 


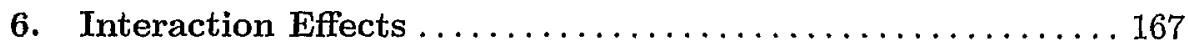

6.1 Diagrammatic Perturbation Theory ................. 170

6.2 Semiclassical Formalism ....................... 171

6.3 Orbital Magnetism of Interacting Diffusive Systems ........ 173

6.3.1 Disordered Rings ........................ 174

6.3.2 Relation to Experiments

and Other Theoretical Approaches............. 176

6.3.3 Two-Dimensional Diffusive Structures ............ 177

6.4 Orbital Magnetism of Interacting Ballistic Quantum Dots ... 179

6.4.1 Ensemble of Squares ....................... 180

6.4.2 First-Order Diagonal Channel................. 181

6.4.3 Renormalization from Higher-Order

Diagonal Contributions ................... 182

6.4.4 Nondiagonal Channel in Regular Systems.......... 184

6.5 Comparison Between Integrable and Chaotic Structures ..... 185

6.6 Comparison with Experiment .................... 187

7. Concluding Remarks $\ldots \ldots \ldots \ldots \ldots \ldots \ldots \ldots \ldots \ldots \ldots$

A. Appendices ............................. 195

A.1 Trace Integrals over Semiclassical Green Functions ........ 195

A.1.1 Auxiliary Integrals ........................ 195

A.1.2 Integrals of Products of Two Retarded Green Functions .............. 198

A.1.3 Integrals of Products of a Retarded and an Advanced Green Function ..... 199

A.2 Numerical Calculations for Susceptibilities

of Square Quantum Dots ...................... 201

A.2.1 Clean Case ............................ 201

A.2.2 Weak Disorder ........................... 202

A.2.3 Thermodynamics........................ 202

A.3 Semiclassical and Quantum Results for Bulk Mean Free Paths 203

References................................. 206

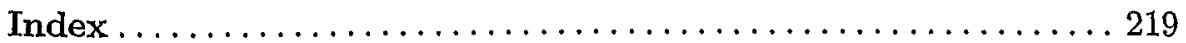

